\title{
Una historia cultural de la Guerra Fría en El Caribe: análisis comparado del nacimiento de los aparatos culturales de Cuba y Venezuela (1960-1980)
}

\section{A cultural history of the Cold War in the Caribbean: comparative analysis of the rise of cultural apparatuses in Cuba and Venezuela (1960-1980)}

\author{
Alejandro Cardozo Uzcátegui \\ Universidad Sergio Arboleda (Colombia) \\ ORCID 0000-0002-4461-3285 \\ alejandro.cardozo@usa.edu.co
}

\begin{abstract}
Resumen
El artículo trata de una historia cultural comparada de la Guerra Fría en El Caribe, de la gestación de los aparatos culturales de Cuba y Venezuela en donde coinciden dos procesos político-culturales nacientes tras la caída, casi en simultáneo, de sus dos dictaduras, por lo que ambos países demandan un nuevo paradigma cultural; a pesar de que en Venezuela nace una democracia y en Cuba una revolución, los cariz culturales de los dos sistemas serán de izquierda revolucionaria, no obstante, en Cuba el aparato cultural germinó desde la revolución para estancarlo en la conservación del régimen del partido, y en Venezuela nació desde el poder establecido pero se le obsequió a intelectuales, artistas y promotores culturales de izquierda para que lo construyeran y desarrollaran en aras de la "Paz democrática" y por la inclusión ideológica de la democracia. Fueron dos líneas diferentes: de la revolución para solidificar el poder establecido (Cuba), y desde el poder establecido para promover la pacificación del país con el costo de abrir los espacios del Estado a una estética revolucionaria de izquierda que, paradójicamente, lucha contra el mismo poder que le dio vida a ese aparato cultural (Venezuela). Como demostramos en el artículo, hubo una simbiosis en ambos modelos, pero con fines estratégicos opuestos.
\end{abstract}

\section{Palabras Clave}

Guerra Fría cultural; Cuba; Venezuela; Democracia, Revolución.

\begin{abstract}
The article deals with a comparative cultural history of the Cold War in the Caribbean, of the gestation of the cultural apparatuses of Cuba and Venezuela, where two emerging political-cultural processes coincide after the fall, almost simultaneously, of their two dictatorships, by what both countries demand a new cultural paradigm; Despite the fact that a democracy is born in Venezuela and a revolution in Cuba, the cultural aspects of the two
\end{abstract}

Esta obra está sujeta a la Licencia Reconocimiento-NoComercial-CompartirIgual 4.0 Internacional de Creative Commons. http://creativecommons.org/licenses/by-nc-sa/4.0/ 


\section{Alejandro Cardozo Uzcátegui}

systems will be of the revolutionary left, however, in Cuba the cultural apparatus germinated since the revolution to stagnate it in the preservation of the party regime, and In Venezuela it was born from the established power but it was given to intellectuals, artists and cultural promoters of the left so that they could build and develop it for the sake of "Democratic Peace" and for the ideological inclusion of democracy. They were two different lines: from the revolution to solidify the established power (Cuba), and from the established power to promote the pacification of the country at the cost of opening the spaces of the State to a revolutionary leftist aesthetic that, paradoxically, fights against the same power that gave life to that cultural apparatus (Venezuela). As we demonstrated in the article, there was a symbiosis in both models, but with opposite strategic ends.

\section{Keywords}

Cultural Cold War; Cuba; Venezuela; Democracy; Revolution.

\section{Introducción}

La proximidad geográfica, entorno geopolítico, vínculos históricos y culturales, son elementos que tienen en común Cuba y Venezuela. La historia contemporánea volverá a unir sus procesos e intereses desde los derrocamientos del dictador cubano Fulgencio Batista (1959) y el venezolano Marcos Pérez Jiménez (1958) en los dos países. Tras el final de ambas dictaduras, nacerán nuevos paradigmas culturales en Cuba y Venezuela, promovidos por la necesidad de crear una nueva cultura política en las nacientes revolución cubana y democracia venezolana.

Lo que para Venezuela será el proceso de democratización más importante de su historia, para Cuba será la revolución castrista sobre la marcha de un rápido giro marxista-leninista radical (Farber, 2010), con lo cual los derroteros políticos de La Habana y Caracas se bifurcarán. Esto último no implica una desconexión de los procesos. En el presente artículo analizaremos la construcción de los aparatos culturales de Cuba y Venezuela, ambos nucleados fuertemente en la estética de izquierda pero de naturalezas diferentes, el primero se encamina hacia el objetivo de construir un férreo establishment cultural alrededor del partido (Guzmán, 2012), y el segundo, aunque auspiciado por el Estado y la democracia, será de naturaleza subversiva, en contra, precisamente, de su benefactor.

En una dimensión comparada podemos dar cuenta de dos hechos: en el Estado revolucionario socialista cubano se va a dar una estética política de izquierda similar al aparato cultural venezolano, no obstante, no va a tolerar desviaciones de la narrativa revolucionaria -revisionismo, crítica, disenso- (Lanza, 2018: 59-60); en cambio, el aparato cultural venezolano, fuertemente inspirado y promovido por la revolución cubana, cabrá cualquier posición política gracias a la democracia mandante (Liscano, 1976).

Daremos cuenta sobre el nacimiento del aparato cultural cubano -desde la mirada suspicaz de los archivos estadounidenses-, la vida política y el relato de alguno de sus protagonistas para demostrar su progresivo compromiso disciplinario con el Estado revolucionario (Lanza, 2018: 36). En Venezuela explicaremos el nacimiento de su aparato cultural como una medida de apertura de espacios alternativos, por 
parte del Estado, a las antiguas militancias guerrilleras y su narrativa y estética revolucionaria. El contrasentido será que en Venezuela la fórmula de convivencia y paz será abrir estos espacios culturales de tolerancia, mientras que en Cuba, la misma narrativa ideológica revolucionaria será represiva (Lanza, 2018: 61).

En Cuba el Estado auspiciará su aparato cultural como un arma de poder blando, este entendido en palabras de J. Nye como "la capacidad de organizar la agenda política de forma que configure las preferencias de otros. La capacidad de marcar preferencias tiende a asociarse con resortes intangibles como una cultura, una ideología y unas instituciones atractivas" (2003: 30); en otras palabras, un sistema de propaganda y diplomacia pública para promocionar la revolución cubana, en donde el mismo aparato será represivo y vigilante (Calduch, 1993); en Venezuela, su aparato cultural será auspiciado por la democracia como antídoto antirrevolucionario: apaciguar el brío guerrillero de la juventud, a los viejos partisanos y la militancia izquierdista universitaria y urbana, en los centros culturales y universitarios que el Estado petrolero puede subsidiar, en un ambiente de democracia y tolerancia ideológica (Massiani, 1977).

La paradoja será que la narrativa ideológica de los intelectuales y movimientos culturales hegemónicos del aparato cultural venezolano propugnan una revolución -inspirada en la cubana- para Venezuela, y será el mismo Estado venezolano quien costeará los medios culturales para esa narrativa estética y política (universidades, editoriales, compañías de teatro, cinematográficas, todas empresas del Estado), mientras en Cuba será impensable que el Estado financie un aparato cultural donde su narrativa estética-política contravenga al Partido, a la nomenclatura ni al gobierno revolucionario, especialmente después de 1972, cuando la revolución ya se encontraba fosilizada: este proceso iría desde la etapa revolucionaria plena en 1959-1961 (con la proclamación del cariz socialista de la revolución en 1961), donde, tras un breve período de libertad creativa, se toman rápidamente las medidas para la transformación al estado socialista, que terminan por reprimir esa incipiente liberación revolucionaria de 1959. Ya en 1972 se crea la Comisión de Estudios Jurídicos del Comité Central del Partido Comunista de Cuba, para crear las bases jurídicas de la Constitución socialista de 1976. Así que el aparato cultural cubano transita desde la "efervescencia creativa" de los momentos iniciáticos de la revolución a la institucionalización postotalitaria sovietista de finales de los sesenta (Rojas, 2015, 131, 181).

\section{Nacimiento del aparato cultural revolucionario cubano Los cuadros fundadores}

El aparato político-cultural cubano nació el mismo día de la Revolución triunfante. Un informe de inteligencia enviado desde la embajada de Estados Unidos en Cuba, del 14 de abril de 1959, por el ministro consejero y encargado de negocios Daniel M. 


\section{Alejandro Cardozo Uzcátegui}

Braddock $^{1}$, da cuenta del origen de este aparato cultural, el más robusto de la región en cuanto a su intención e impacto ideológico. El funcionario identificó algunos personajes y métodos, los cuales evolucionaron prodigiosamente en el uso de estrategias "blandas" para la acción cultural con el fin de profundizar el proceso político y lograr la exportación y traslación ideológica al resto de América Latina.

El documento admite que los militantes comunistas cubanos "han sido tradicionalmente fuertes en el campo de las actividades culturales", ni siquiera durante la dictadura de Batista cesaron el activismo político-cultural. Se identifica al Instituto Nacional de Cultura como el principal grupo cultural nacional en Cuba, encabezado, menciona el informe, por Vicentina Antuña Tavío, considerada como "una compañera de viaje" (Braddock, 1959). Durante la lucha contra Batista, Antuña Tavío (1909-1993), docente de Lenguas Clásicas (latinista) e intelectual cubana, fue cercana al Movimiento 26 de Julio como responsable de la "célula S" de la Resistencia Cívica. Antes de la Revolución fue militante del Partido del Pueblo Cubano (Ortodoxo). Depuesto Batista fue directora de Cultura del Ministerio de Educación y más tarde presidenta del nuevo Consejo Nacional de la Cultura. Luego asumió la dirección de la Escuela de Letras de la Universidad de La Habana y la presidencia de la Comisión cubana de la Unesco (Alarcón, 2009). Fundadora y secretaria del Movimiento por la Paz y miembro del Consejo Mundial por la Paz (Miranda, 2009), uno de los organismos de acción cultural de la URSS. Roberto Fernández Retamar, influyente intelectual cubano, fue discípulo de Antuña Tavío. Esta erudita cubana, revolucionaria y comprometida con el proceso de creación cultural como un factor para la Guerra Fría, es uno de los baluartes en la configuración de este dispositivo de traslación ideológica. Decía Antuña que había que incentivar la lectura, "cultivar los valores estéticos e ideológicos importantes para elevar la cultura de nuestro pueblo" (Antuña 1985, 220). Antuña Tavío influyó sobre una generación entera de humanistas cubanos, y desempeñó una actividad diplomática en el orden cultural para promocionar la revolución cubana en foros mundiales.

En abril de 1959 la Junta de Gobierno del Instituto Nacional de Cultura aún no había sido seleccionada. Plantea el informe Braddock que la estructuración del Instituto "presumiblemente indicará la coloración política de esta organización". El ministro de Educación cubano "ha designado una comisión para reescribir los textos de la historia cubana. En esta comisión de nueve hombres están el Dr. Antonio Núñez Jiménez, reportado como pro-comunista, y el Dr. Elias Entralgo, considerado en la categoría de compañero de viaje" (Braddock, 1959). Antonio Núñez Jiménez (19231998), el "cuarto descubridor de Cuba" (título honorífico otorgado por la Sociedad Espeleológica de Cuba y la Sociedad Cubana de Geografía) es considerado "el padre" de la Espeleología Cubana. Fue el primer presidente de la Academia de Ciencias de Cuba y presidente fundador de la Federación Espeleológica de América Latina y el Caribe. Durante la Revolución fue nombrado capitán del Ejército Rebelde y estuvo

1 Último funcionario diplomático estadounidense en salir de Cuba, una vez rotas las relaciones de la isla con Estados Unidos en 1961 (Chao, 2019). 


\section{Una historia cultural de la Guerra Fría en El Caribe: análisis comparado del nacimiento de los aparatos culturales de Cuba y Venezuela (1960-1980)}

bajo las órdenes del Che Guevara. Después de la caída de Batista fue director ejecutivo del Instituto Nacional de Reforma Agraria y presidente de la comisión redactora de la radical Ley de Reforma Agraria cubana. Como capitán ayudante del Che Guevara en la fortaleza militar de La Cabaña, tuvo que ser testigo de los fusilamientos a los disidentes revolucionarios consumados en la fortaleza.

Entre los años 1960-1961 Núñez dirigió la Escuela de Artillería Camilo Cienfuegos para entrenar a 10.000 milicianos. Fundador del Partido Comunista de Cuba en 1965. Por instrucciones de Fidel, fue miembro y creador de la primera Milicia Campesina de Cuba, organización de seguimiento y captura de células disidentes en la Sierra de los Órganos. En 1960 fue el primer embajador oficial de Cuba en la Unión Soviética, y en lo sucesivo, lo sería en casi todos los países del Pacto de Varsovia. Tuvo una agitada vida diplomática en los foros de exportación ideológica cubana durante la Guerra Fría; uno de los funcionarios principales de la delegación del viaje de Fidel a la ONU en 1960; delegado de Cuba al XX Aniversario de la Victoria Contra el Fascismo en Moscú; representante cubano para el XXVI Congreso del Partido Comunista de la Unión Soviética, en Moscú; presidió la delegación cubana para el primer aniversario de la independencia de Argelia, y el mismo cargo en la misión cubana para celebrar el primer aniversario de la liberación de Kampuchea; fue fundador de la Comisión Nacional Cubana de la Unesco (EcuRed, 2020).

Núñez representa el ideal socialista del intelectual "orgánico", es decir, aquel que marida la academia y la política de forma prolija, pues sobrevive y triunfa en las dos escenas. Fue invitado por el servicio meteorológico de la URSS para la expedición soviética al Polo Norte de 1972, y una década más tarde participó en la $27^{\circ}$ expedición soviética a la Antártida. Prolífico autor y documentalista de cine. El informe de Braddock lo ubica entre los personajes claves de la temprana estructuración de este complejo aparato político-cultural cubano.

Asimismo, este informe habla de la organización cultural del frente comunista "Nuestro Tiempo", organización clandestina entre 1957-1958, que tras el triunfo de la Revolución se activó, y tuvo entre sus primeros oradores al Che Guevara. Los programas culturales en la fortaleza de La Cabaña, informa Braddock, han incluido jornadas de alfabetización y una "inyección de marxismo, así como lecturas de poesía de Nicolás Guillén, destacado poeta comunista cubano". Se relata en el informe que el poeta Guillén declamó su poema "Little Rock" sobre la discriminación racial² en los Estados Unidos (Braddock, 1959).

También hay noticias de la actividad comunista entre los estudiantes de la Universidad de La Habana. Se señala que el activismo político filocomunista entre los estudiantes es todavía escaso. Informa sobre una masiva concentración estudiantil hace dos meses, donde uno de los estudiantes pronunció un discurso de línea comunista. Sin embargo, para medir el alcance de la actividad comunista en la

\footnotetext{
2 Consta en la documentación especializada que la solidaridad internacional con las víctimas del racismo estaba entre los lineamientos principales del Politburó soviético (Zubok, 2008: 506).
} 


\section{Alejandro Cardozo Uzcátegui}

Universidad de La Habana, habría que esperar durante los próximos meses, cuando la Federación de Estudiantes celebre elecciones (Braddock, 1959).

La incipiente actividad entorno a la cinematografía también es observada en el informe, cuando advierte sobre Cine Club-Visión, donde se tiene noticias de una intervención hecha por el "geógrafo procomunista cubano Dr. Antonio Núñez Jiménez". El programa Cine-Debate trata de la proyección de una película seguida de un debate con la audiencia. La entrada es de veinte centavos. Una de las primeras proyecciones se llevó a cabo en la sección de trabajadores de Santos Suárez, en La Habana. "Si bien no se ha reportado ninguna evidencia de actividad comunista en el Cine-Debate, se entiende que esta técnica es a veces utilizada por los comunistas". Juan Marinello, líder intelectual comunista, retornó como maestro en la Escuela Normal de La Habana, en enero. Al poco tiempo voló a Moscú, donde se sabe que dio una charla sobre José Martí. Asimismo, habla de la televisión cubana, específicamente de dos programas recientes dedicados a debates entre comunistas y católicos, donde se evidencia "la respetabilidad de la doctrina comunista como doctrina actual" (Braddock, 1959).

Juan Marinello Vidaurreta (1898-1977) abogado, poeta y ensayista cubano, fue miembro del Partido Socialista Popular, el partido de militancia de Raúl Castro y el mismo partido que envió delegados a Moscú para convencer a la nomenclatura soviética sobre el cariz marxista-leninista de la Revolución cubana. Podríamos inferir que, por ende, el poeta Marinello Vidaurreta fue cercano a Raúl Castro por la militancia en el PSP antes de la Revolución. Una vez que triunfan los Castro, Marinello Vidaurreta fue rector de la Universidad de La Habana, también embajador de Cuba en la Unesco. Fue miembro de la nomenclatura cubana: de la Mesa Constitutiva de la Asamblea Nacional del Poder Popular, miembro del Comité Central del Partido Comunista de Cuba y del Consejo de Estado hasta su fallecimiento; fue, por ende, de los intocables padres de la Revolución, presidió también la Sociedad Cubano-Mexicana de Relaciones Culturales, un organismo muy activo en la irradiación político-cultural de los valores de la Revolución (EcuRed, 2020). Así también el poeta Marinello Vidaurreta fue presidente del Movimiento Cubano por la Paz y Soberanía de los Pueblos, franquicia del Consejo Mundial por la Paz, que como hemos acotado, uno de los órganos más dinámicos de acción políticocultural soviética. Se señala en el informe que el "tema del anticomunismo como parte integrante de la línea política de Batista y como elemento contrarrevolucionario, fue tratado por Juan Marinello en una editorial en Hoy, a principios de enero" (Braddock, 1959).

La embrionaria organización de un aparato editorial revolucionario también es observada por funcionarios de la embajada de Estados Unidos en Cuba. Son testigos del nacimiento de uno de los más dinámicos sistemas editoriales dentro del aparato cultural revolucionario, como es el cubano. El desempeño ideológico de periódicos, revistas y suplementos literarios es percibido con preocupación desde la inteligencia estadounidense pues, como hemos acotado antes, la verdadera -o más 


\section{Una historia cultural de la Guerra Fría en El Caribe: análisis comparado del nacimiento de los aparatos culturales de Cuba y Venezuela (1960-1980)}

eficiente- cadena de transmisión política de la Guerra Fría fue dada a través de estos sistemas de agitación editoriales, escriturales, artísticos y en general, culturales.

Los analistas de la embajada estadounidense en Cuba, en abril de 1959, informan que buena parte de la industria editorial (linotipias, talleres de prensa, salas de prensa y departamentos administrativos de periódicos) está en manos del Sindicato de Artes Gráficas, "una organización dirigida por los comunistas". En este informe de inteligencia se detectaron como publicaciones del engranaje ideológico -aunque en ocasiones de forma solapada- del ala comunista del gobierno cubano a los periódicos y revistas siguientes: Hoy; Revolución con su suplemento cultural "Lunes de Revolución" (el 6 de abril de este año, advierte Braddock, el suplemento publicó entero el Manifiesto Comunista de Marx); la revista comunista Mella, Fundamentos y Carta Semanal.

"El Gobierno parece estar avanzando en la dirección de una toma de control en el área clave de la información pública, y hay evidencia considerable de infiltración comunista". Identifican a Carlos Franqui, Paco Alfonso y David Salvador Manso como los operadores ideológicos de esta "toma de control" de medios de comunicación. Reconocen de primera mano a Carlos Franqui (1921-2010), fundador y editor en 1959 de Revolución, peligroso agente cultural "sospechoso en ocasiones de ser comunista y, en cualquier caso, parece compartir muchas ideas comunistas", según anota Braddock. En efecto, la primera militancia juvenil de Franqui fue en el Partido Socialista Popular (fundado en 1925 como Partido Comunista Cubano), joven y convencido ideológico.

Sin embargo, es más que llamativo Franqui por tratarse de aquellos primeros agitadores culturales de la Revolución, piezas esenciales del primigenio engranaje del sistema cultural y propagandístico revolucionario, que acaban en la desconformidad política, actitud no tolerada por el aparato cultural revolucionario. Franqui, en las primeras de cambio revolucionario, fue director de Radio Rebelde, con transmisiones desde Sierra Maestra; miembro temprano del Movimiento 26 de Julio y como se leyó, fundador-editor de Revolución. Sin embargo, más tarde fue un documentado crítico del giro marxista-leninista de la Revolución cubana y de la denominada por Franqui "ideología del poder" de Fidel Castro. Su esclarecida producción intelectual sobre la Revolución cubana puede ser una de las obras fundamentales para el estudio de la Guerra Fría en el Caribe y su asociación con la Unión Soviética, alianza que desmanteló el primer proyecto de Sierra Maestra: la "Carta de la Sierra" y el plan de la restauración de la Constitución de 1940. La transformación revolucionaria para Carlos Franqui fue así: "en la práctica, en el mundo comunista, el Partido sustituye al proletariado; después, el Comité Central sustituye al Partido, y después, el secretario del Partido sustituye al Comité Central, y así se crea el jefe absoluto" (Cayuela, 2006).

El documento de Braddock también señala a Paco Alfonso (1906-1989) a cargo del canal televisivo del gobierno cubano, "canal 12". Se indica su filiación comunista y el peligro inminente de que todo el sistema radio-televisivo cubano quede, finalmente, 


\section{Alejandro Cardozo Uzcátegui}

bajo una sola línea política: Radio COCO, Radio CNC (que es Radio Rebelde) y Radio Unión. Paco Alfonso antes de la Revolución ya era un destacado actor de teatro y dramaturgo. Militante del PSP cubano, y prolífero productor de obras de teatro, cine y televisión. Llevó a la isla la obra de Máximo Gorki, Los bajos fondos e introdujo piezas de la dramaturgia soviética como Los hombres rusos de Simonov e Invasión de Leonov (EcuRed, 2020).

David Salvador Manso controlaba Radio Unión en la primavera revolucionaria del 59. Braddock advierte que por su compromiso ideológico ese control mediático podría ser ruinoso para los espectáculos de La Habana, pues podía haber una censura a la publicidad de estos eventos. David Salvador Manso es otro caso -tanto emblemático como desconocido- de disidencia al régimen de los hermanos Castro. Fue militante del PSP, estuvo en Sierra Maestra en la Dirección Nacional del Movimiento 26 de Julio, y una vez que triunfó la Revolución fue líder del movimiento obrero cubano (secretario general de la Confederación de Trabajadores de Cuba en su X Congreso).

Durante este mismo congreso se opuso a la radicalización del movimiento obrero por la inclusión de varios cuadros comunistas en la CTC. Manso provenía del movimiento sindical organizado por la Compañía de Jesús y la Juventud Obrera Católica, la cual deseaba restar la influencia de los militantes comunistas en la conformación sindical cubana. No obstante, logró su postulación al cargo como presidente de la confederación sindical, era el candidato de Fidel aunque iba en contracorriente (Chaffee, 1992: 8). ¿Tendría relación la formación jesuita de los Castro? ¿0, acaso, era una estrategia disimular en 1959 el elemento ideológico comunista con antifaces de pluralidad en el estratégico movimiento sindical obrero? Manso soportó la presión y su condicionado liderazgo por poco tiempo, pues el 14 de abril de 1960 dimitió a la secretaría general de la CTC y formó un movimiento de choque, el Movimiento 30 de Noviembre (M-30-11) que gozó de cierto apoyo logístico de la CIA (Torreira, 2005: 14).

En noviembre de 1960 David Salvador Manso fue capturado por la policía política cubana y condenado a 30 años de prisión. En un documento desclasificado de la CIA de abril de 1965, explica que mientras estaba encarcelado en la Isla de los Pinos en 1963, Manso fue expulsado del M-30-11. El mismo informe de la CIA advertía que el M-30-11 planeaba con Fidel Castro hacer una "falsa revolución" para satisfacer al pueblo cubano y a los observadores internacionales, liberar prisioneros políticos y toda la farsa se llevaría a cabo confiando el comando del proceso a los mismos cuadros comunistas del gobierno. El eslogan de la revolución sería "Fidelismo sin Fidel", y llevaría a cabo unas elecciones falsas. Llama la atención la estrategia del M30-11, que según la CIA, ya había tenido contacto con el exilio cubano y con la prensa estadounidense. Expulsar del movimiento a Manso era parte de una conspiración de contrainteligencia de mayor alcance, pues el M-30-11 planeaba infiltrarse entre los líderes del exilio cubano, varios partidos anticastristas y en la prensa estadounidense. Finalmente queda dicho en el informe de la CIA que el M-30-11 
había sido tomado por el G-2 cubano y estaba haciendo lobby político y mediático en Estados Unidos y Cuba (CIA, 1965).

\title{
El fin de la utopía cubana comenzó desde 1961
}

Si bien es cierto que 1971 será una fecha paradigmática del fin de la libertad creativa en Cuba, cuando se estableció "la nueva política cultural cubana", (como lo denominó Ángel Ramas en su artículo-manifiesto), el hecho de que la creación artística literaria debía ser un "arma de la Revolución” (Rojas, 2018, 14) era una línea trazada una década antes por Fidel Castro.

Fidel lo planteó con bastante elocuencia en 1961, tempana fecha que algunos no admiten para hablar, todavía, de represión creativa, cuando en efecto, el comandante lo afirma ante los temores de los artistas cubanos de no gozar plena libertad creadora en la revolución, Castro dijo:

\begin{abstract}
¿Cuál debe ser hoy la primera preocupación de todo ciudadano? ¿La preocupación de que la Revolución vaya a desbordar sus medidas, de que la Revolución vaya a asfixiar el arte, de que la Revolución vaya a asfixiar el genio creador de nuestros ciudadanos, o la preocupación por parte de todos debe ser la Revolución misma? ¿Los peligros reales o imaginarios que puedan amenazar el espíritu creador, o los peligros que puedan amenazar a la Revolución misma? (...) Y cabe preguntarse si un revolucionario verdadero, si un artista o intelectual que sienta la Revolución y que esté seguro de que es capaz de servir a la Revolución puede plantearse este problema. Es decir, que el campo de la duda no queda ya para los escritores y artistas verdaderamente revolucionarios; el campo de la duda queda para los escritores y artistas que sin ser contrarrevolucionarios no se sientan tampoco revolucionarios (...) Y el artista más revolucionario sería aquel que estuviera dispuesto a sacrificar hasta su propia vocación artística por la Revolución (Castro, 1961).
\end{abstract}

Como lo describe Quintero Herencia en su libro sobre Casa de las Américas (Quintero, 2002, 67), la revolución exigía al escritor "ser y estar en ella". Casa de las Américas como la misma revista se elaboran en ese espacio que no podía ser otra cosa que revolucionario. Quintero da cuenta de esa sistemática inflexibilidad y bruma política que tuvo lugar en Cuba entre los años de 1968 a 1971. Sin embargo, las líneas ya habían sido trazadas por el líder supremo de la revolución desde junio de 1961 en sus "Palabras a los intelectuales" en la Biblioteca Nacional: fuera de la revolución no se admitiría nada, y estar fuera, podía ser simplemente disentir o criticar la más mínima forma del proceso revolucionario.

\section{La revista Bohemia y el castigo por disentir con la Revolución}

Había indicios de que los líderes en el campo de la información como Bohemia y las estaciones CMQ y Telemundo, se expresarían rotundamente en 1959 contra la infiltración comunista y la autocensura del gobierno castrista si obtuvieran más 


\section{Alejandro Cardozo Uzcátegui}

"apoyo moral" de Estados Unidos y en particular de su embajada en Cuba, con "garantías de que nuestra posición anticomunista se aclare a los líderes de las autoridades del gobierno cubano". Buena parte de "la prensa responsable no simpatiza con el comunismo, pero el anticomunismo se ha identificado con tanto éxito con el pro-americanismo, que en este momento es impopular debido a los continuos ataques de Fidel Castro contra los Estados Unidos", incluso los escritores anticomunistas titubean en tomar posiciones firmes respecto a la ideología reinante. Además, continúa el informe, debido a las obvias similitudes entre el programa comunista y el programa revolucionario, los críticos del comunismo "corren el riesgo de ser llamados contrarrevolucionarios y encarcelados, y pocos de ellos desean ser héroes" (Braddock, 1959).

El informe señala el rol de Bohemia en estas preliminares de la Guerra Fría cultural, como un órgano informativo que esperaba apoyo de la embajada de Estados Unidos para pronunciarse en contra del irreversible proceso ideológico que estaba sufriendo la isla. Es sugerente que salga a colación la revista Bohemia, pues su propietario y director, Miguel Ángel Quevedo (1908-1969), será un protagonista y una víctima del sistema cultural de la Guerra Fría latinoamericana.

Bohemia fue una de las publicaciones más influyentes de Cuba y América Latina. Atacó al régimen de Batista y apoyó la Revolución cubana; el 26 de julio de 1958 la revista publicó entero el "Manifiesto de Sierra Maestra”, y en poco menos de un año, el 11 de enero de 1959, la tirada de un millón de ejemplares del número especial "Edición de la libertad" de Bohemia con una imagen de Fidel Castro que rezaba "Gloria al Héroe Nacional" se vendió por completo y en pocas horas. No obstante, ¿por qué Braddock la identifica como una publicación dispuesta a denunciar la infiltración comunista y la autocensura del castrismo tras semejante apoyo? En una carta que nos atrevemos a denominar "la epístola de la Guerra Fría latinoamericana", Miguel Ángel Quevedo le escribe desde Caracas (12 de agosto de 1969) al poeta cubano Ernesto Montaner (exiliado en Miami), como acto de contrición política, sobre la culpa que le embarga haber usado a Bohemia como medio de propaganda del castrismo durante aquella primavera revolucionaria: "Fidel no es más que el resultado del estallido de la demagogia y de la insensatez. Todos contribuimos a crearlo. Y todos, por resentidos, por demagogos, por estúpidos o por malvados, somos culpables de que llegara al poder. Los periodistas que conociendo la hoja de Fidel, su participación en el Bogotazo Comunista, el asesinato de Manolo Castro y su conducta gansteril en la Universidad de la Habana, pedíamos una amnistía para él y sus cómplices en el asalto al Cuartel Moncada" (Quevedo [1969], 2019). La carta continúa señalando a los culpables de que el castrismo tomara el poder absoluto en Cuba, culpabiliza desde la iglesia cubana por enviar jóvenes a la Sierra Maestra para "servir" a Castro, hasta al mismo gobierno de Estados Unidos por incautar las armas para derrocar a los "guerrilleros", aquí tal vez se refiere Quevedo a Bahía de Cochinos.

Sobre el mea culpa del papel de Bohemia en aras del proyecto castrista, escribe

"Bohemia no era más que un eco de la calle. Aquella calle contaminada por el odio 
que aplaudió a Bohemia cuando inventó «los veinte mil muertos». Invención diabólica del dipsómano Enriquito de la Osa [quien más tarde Fidel nombra director de la revista], que sabía que Bohemia era un eco de la calle, pero que también la calle se hacía eco de lo que publicaba Bohemia" (Quevedo [1969], 2019). Los "veinte mil muertos" fue una falsa noticia de las supuestas víctimas del régimen de Batista, un número inflado con propósitos propagandísticos que se volvió una consigna: “iParedón! ¡Paredón! ¡Rusia si, yanquis no! ¡Si Fidel es comunista, que me pongan en la lista!, y repetían como sirenas «los 20.000 muertos de Batista»" (Torricella, 2012). También señala Quevedo su estado de soledad, tanto existencial como política: "Muero asqueado. Solo. Proscrito. Desterrado. Y traicionado y abandonado por amigos a quienes brindé generosamente mi apoyo moral y económico en días muy difíciles. Como Rómulo Betancourt, Figueres, Muñoz Marín. Los titanes de esa «Izquierda Democrática» que tan poco tiene de «democrática» y tanto de «izquierda». Todos deshumanizados y fríos me abandonaron en la caída. Cuando se convencieron de que yo era anticomunista, me demostraron que ellos eran antiquevedistas. Son los presuntos fundadores del Tercer Mundo. El mundo de Mao Tse Tung" (Quevedo [1969] 2019). Esta epístola de la Guerra Fría es también donde Quevedo anuncia al poeta Montaner que "al fin" se suicidará, y ese mismo 12 de agosto lo hace. Nueve años antes, el 17 de julio de 1960, Quevedo congregó a los periodistas y empleados en la redacción cubana de Bohemia para anunciar su viaje al exilio. Se llevó consigo parte del equipo editorial para fundar en Nueva York Bohemia Libre, que terminó editándose en Venezuela (Cancio, 2008; Domingo, 2007: 114).

Bohemia fue una de las revistas más influyentes en la cultura política latinoamericana ${ }^{3}$. Una vez que Quevedo se exilia (primero a Estados Unidos, luego a Venezuela) el castrismo secuestra la revista, tanto su representación histórica como simbólica, transformándola en "la traducción popular de la ideología del gobierno socialista de Castro" en donde, sin embargo, aún guardaba ciertas características originarias (Lambert 1996, 133). Es hacia los años 80 cuando llega a Bohemia Martha Harnecker con otra propuesta de trabajo editorial. Harnecker, conocida por sus manuales iniciáticos de materialismo histórico dialéctico, era esposa de Manuel Piñeiro, alias Barba Roja, jefe del Departamento América del Partido Comunista de Cuba. En funciones, fue el principal organizador del apoyo cubano a las guerrillas latinoamericanas (Ruiz 2003, 51). No se puede afirmar que la llegada de Harnecker coincidiera -o estimulara- con la fase editorial más decadente y pobre de Bohemia, sin embargo, aquí también queda como prueba o adosando otro ejemplo, la convergencia política de la cultura de izquierda con grupos de formación ideológica, los movimientos guerrilleros y la exportación de esa eficiente maquinaria cubana de propaganda durante la Guerra Fría latinoamericana.

El hecho que la revolución cubana en su propuesta rupturista o vanguardista -en lo ideológico como en lo cultural- se haya estancado por la institucionalización

${ }^{3}$ Fundada por Miguel Ángel Quevedo (padre) en 1908. 


\section{Alejandro Cardozo Uzcátegui}

sovietista (1972-1976), no quiere decir que haya cesado su ímpetu propagandístico y su dinámica de diplomacia pública (Slack y Whitson, 1988).

\section{Venezuela y su aparato cultural: desde el Estado contra el Estado El cine}

El cine como arte y como industria con fines políticos-culturales, puede ser de las armas más eficientes en todo sistema de propaganda y está en todos los aparatos culturales. En Estados Unidos, por ejemplo, corría el año de 1956 cuando miembros del Estado Mayor Conjunto del Ejército y la CIA establecieron una hoja de ruta para modelar la producción cinematográfica hacia un discurso anticomunista, procurando distanciarse de los problemas sociales locales de ese momento como el racismo y los derechos civiles. Se hicieron reuniones con la Metro en Hollywood contando con el apoyo de Ward Bond, John Wayne, Merian Cooper, John Ford, entre otros (Fontana, 2013).

Asimismo, para los bolcheviques el aporte del cine al aparato cultural soviético era de los más importantes. Le escribió Lenin en 1919 a Lunatcharsky, comisario del pueblo para las Bellas Artes: "Cuando mejore la situación general del país recibirá usted algunos créditos para que aumente su producción y, sobre todo, para que haga penetrar el cinematógrafo en las masas. Debe usted tener en cuenta que de todas las artes, la más importante para nosotros es precisamente el cine" (Untermeyer, 1957). El cine en Venezuela, como en la mayoría de los países, no es una industria autosustentable y por ende requiere de una fuerte participación del Estado. La industria cinematográfica venezolana ha sido enteramente de capital público, del Estado y, sin embargo, su producción durante la Guerra Fría cultural venezolana fue toda prácticamente de izquierda.

Jesús Enrique Guédez, pionero del cine documental venezolano y primer presidente de la Asociación Nacional de Autores Cinematográficos (ANAC) formó en 1968 el grupo Cine Urgente, propuesta amasada en su experiencia en Italia y en el documentalismo europeo para plantear el tema social, desde el prisma de la izquierda revolucionaria, basados en hechos coyunturales. Dijo en entrevista Guédez que "quienes desarrollaron este tipo de cine, fue gente de clase media de grupos intelectuales y militantes de los movimientos políticos de izquierda" (Guédez, 2013). Una muestra de este cine políticamente cooptado por la intelectualidad del Partido Comunista Venezolano (y de sus posteriores derivas como el Movimiento al Socialismo) es "Pozo Muerto" (1967) de Carlos Rebolledo y Edmundo Aray, "La Ciudad que nos ve" (1967) de Guédez y Josefina Jordán, y muestras anteriores como "Caín adolescente" (1959), ópera prima de Román Chalbaud y la premiada "Araya" (1959) de Margot Benacerraf. La muestra se extiende por toda la década de un cine ciertamente revolucionario: "La Universidad Vota en Contra” (1968), "Renovación” (1969), “22 de Mayo” (1969), “Estallido” de Nelson Arrieti; “Sí Podemos” que planteó la búsqueda de Cine Urgente y el grupo del 
MAS, donde trabajaron Josefina Jordan, Jacobo Borges, Perucho Laya, Franca Donda (Acosta, 1997).

Casi la totalidad de la propuesta cinematográfica venezolana estuvo ganada hacia la denuncia social, la cotidianidad de la violencia política, la lucha guerrillera y la narrativa insurgente, es decir, fue un cine desde la izquierda y para la izquierda en contra del establishment venezolano. Sin embargo, el estado auspició todas las obras referidas a través de la empresa cinematográfica Bolívar Films, la Cinemateca Nacional, entre otras subsidiarias, con fuerte participación de capital estatal (Káiser, 2011: 631-641).

\section{Los escritores y los grupos culturales}

A finales de la década los años cincuenta en París, en el exilio, los poetas Alfredo Silva Estrada, Alfredo Chacón y Roberto Guevara, editan Señal (Cardozo, 1976), revista germen de Sardio, publicación homónima del grupo literario (Arráiz, 2003). La evolución de Sardio recoge peregrinamente la dinámica impuesta por la Guerra Fría. El grupo, constituido poetas y pintores, sufrirá la escisión de la Venezuela intelectual que se compartimenta -más que adscribirse- en dos visiones del país político e ideológico que impone la Guerra Fría cultural, pues en 1961, tras la confesión comunista de Fidel Castro en Cuba se dividen las aguas de Sardio. El primero en recogerse fue Guillermo Sucre, quien como anota Arráiz (2003), era el teórico del grupo. Aunque Sardio se compacta más por su propuesta estética "reminiscencias venezolanas -voces, vivencias, paisajes (...) tratados con un nuevo lenguaje, con un nuevo aire, con anhelos universales..." (Cardozo, 1976: 67) el argumento ideológico desquebrajó todo lo demás.

Del grupo anterior hubo quienes apostaron por el país democrático que ofrecía el establishment, no veían en la radiación cultural-ideológica de Cuba otra cosa más allá de un socialismo real, una nueva expresión del espíritu autoritario del que habían padecido en las dictaduras que antecedieron a la naciente democracia venezolana. Unos y otros habían vivido la prisión, el exilio -o la clandestinidad-impuestos por el perezjimenato, y no pocos habían sufrido la dictadura del gomecismo (19081935). Otro autoritarismo, o en el mejor de los casos, otro dogmatismo era intolerable aquella parte de Sardio que se vincularía al proyecto democrático del proemio democrático de Puntofijo (1958-1964).

Los miembros del alero de izquierda de Sardio, desencantados de la democracia representativa que daba sus primeros pasos en el gobierno de Rómulo Betancourt (1959-1964), pensaron que las ideas concebidas durante la transición de la dictadura al régimen naciente, empezaban a ser burladas. Sin embargo, señala Cardozo que la revista Sardio -junto a Tabla Redonda- “...aparecen en un momento muy peculiar de la reciente historia política venezolana, ha sustituido a la dictadura perezjimenista el gobierno transicional de Larrazábal. Breve época de optimismo, y 


\section{Alejandro Cardozo Uzcátegui}

de violenta transición hacia la larga noche del betancourismo. Como esos años de Larrazábal, así fueron Sardio y Tabla Redonda, revistas de transición" (1976: 68). Ese movimiento, probablemente transicional, además de reinventarse más tarde en otras tendencias, abre el camino a la narrativa insurgente de izquierda. Así el primer editorial de la revista, donde aclaran el compromiso ideológico de este tipo intelectual frente al contexto político venezolano:

Ser artista implica tanto una voluntad de estilo y un ejercicio del alma como reciedumbre moral y un compromiso ante la vida (...) Es menester quemarse un tanto en el fuego devorante de la historia (...) Ser político equivale a tanto como ser hombre. Toda indolencia es propicia a la esclavitud y a la humillación del espíritu (...) por ello es que cultura y tiranía son radicalmente incompatibles (Arráiz, 2003: 215).

Compañera de Sardio -en la cronología- será la revista Tabla Redonda, donde a diferencia de la primera, había el propósito de "denunciar con voz revolucionaria la realidad venezolana" (Cardozo, 1976: 67). Los miembros de Tabla Redonda militaron en el Partido Comunista de Venezuela (PCV). La lucha contra la última dictadura les da los argumentos para ahora ser militantes de la izquierda antisistema venezolana. El argumento estético, poético y ensayístico se une a la doctrina comunista en el contexto político venezolano. En la plana mayor de Tabla Redonda destacan las figuras de Rafael Cadenas, Arnaldo Acosta Bello, Caupolicán Ovalles, Samuel Villegas, Jesús Sanoja Hernández, Jesús Enrique Guédez y Ángel Eduardo Acevedo. Personajes notables en la poesía nacional como José Pepe Barroeta orbitaron alrededor de Tabla Redonda, pero tendrán mayor protagonismo en otros grupos culturales como el más conocido de todos, Techo de la Ballena.

Tabla Redonda apoyó de forma amplia y convencida la lucha armada guerrillera de las Fuerzas Armadas de Liberación Nacional, que eran la unión guerrillera de la militancia del Partido Comunista de Venezuela y el Movimiento de Izquierda Revolucionaria. El movimiento guerrillero tuvo una fuerte resonancia en esta "poética” de Guerra Fría (Aray, 2017: 44-45).

El cineasta venezolano Edmundo Aray, en su poemario Tierra roja, tierra negra (primera edición de 1968) plantea buena parte de la fenomenología de la Guerra Fría: el fundamento antiimperialista de la doctrina tercermundista devenida del artículo de Alfred Sauvy "Trois mondes, une planète”, la crisis en Estados Unidos de los derechos civiles, para más adelante unirse con el movimiento antibelicista durante la guerra de Vietnam. El poema aduce a esta guerra como referente para el resto de los pueblos. Este poemario está tan encajado en la atmósfera de la Guerra Fría, que rebusca en elementos cotidianos de ese momento histórico -que entonces son la noticia cotidiana- como el período de mayor recrudecimiento del conflicto en Indochina en la administración de Lyndon Johnson, el período de "norteamericanización" de la guerra durante la fase denominada la War of Attrition, la guerra de agotamiento en el poema "Conocí a un hombre" (Aray, 2017: 55-56). Sugiere el poema a "mis infames bombas... mis raudos aviones... mis amados ángeles 


\section{Una historia cultural de la Guerra Fría en El Caribe: análisis comparado del nacimiento de los aparatos culturales de Cuba y Venezuela (1960-1980)}

del mar y del aire... ¡los alados!" evocando con ironía la operación Rolling Thunder, piedra angular de la aviación estadounidense de localización de objetivos y bombardeo sobre Vietnam del norte y el camino de Ho Chi Minh.

Edmundo Aray fue un poeta cercano a Sardio y al Techo de la Ballena. La revista Sardio (1958-1961) publica sus primeros textos sobre cine. Como integrante embrionario del movimiento Techo de la Ballena -al que volveremos enseguidaAray le transfirió un vigor especial (que lo hace perdurable) a un movimiento signado, tal vez, a ser efímero por la insolvencia intelectual de su propuesta, atada realmente a la admiración del fenómeno revolucionario cubano.

El panorama político para muchos es más complicado y sinuoso de lo que se imaginaron en la transición. La lucha gubernamental contra los focos de insurgencia de la izquierda militarmente organizada -campo y ciudad-arrecia. Sardio, en palabras de Cardozo "se revienta" y, como se ha acotado, muchos de sus integrantes escogen la vía en contra del sistema establecido, de la democracia representativa venezolana, y donde buena parte de ellos serán fundadores de El Techo de la Ballena. Este grupo prolífero y variopinto de poetas, ensayistas, narradores, pintores y cineastas ${ }^{4}$ estaba encabezado -debido a la notoriedad de una obra artística integralpor Juan Calzadilla.

El mismo Calzadilla, en una entrevista de 1995, delibera sobre el movimiento El Techo de la Ballena y condensa nuestra propuesta: fue movimiento que nació y creció por las bondades del sistema político venezolano que financiaba y toleraba la disidencia y narrativa procastrista, revolucionaria y socialista; sin embargo, en el fondo, sus miembros y militantes sabían que era solo un divertimento, que en el fondo era una actitud que solo una democracia sobrellevaría: "Curiosamente fue un movimiento subversivo que, aun apoyando la revolución y tomándola en serio, nunca creyó en el éxito de la subversión" por tanto, dice Calzadilla, "Sabíamos que si el socialismo llegaba a implantarse en el país, como resultado de la lucha armada inspirada en Castro, perderíamos enseguida esas mismas libertades en nombre de las cuales se luchaba" (1995: 24-28).

En la década de los sesenta las publicaciones siguen emergiendo con exaltación izquierdista, muy cercanos a la revolución cubana, más que a la Unión Soviética. La cuestión se centra con preferencia en poesía y narrativa. Tras Sardio, Tabla Redonda, Rayado sobre el techo, surge Sol cuello Cortado dirigida en la práctica por Caupolicán Ovalles y Ludovico Silva, este último un prolífico filósofo marxista (Calles-Santillana, 2013). Asimismo Letra Roja donde destaca Jesús Sanoja Hernández como colaborador frecuente en este suplemento literario de corta existencia (1963). Lam (escribieron ahí los poetas Mary Lu Sananes y López Sanz). La publicación más influyente en ese río fue En Haa (1962) dirigida por Teodoro Pérez Peralta y Lubio Cardozo. Decimos influyente por dos peculiaridades: sobrevivió el torbellino de los años sesenta y sus páginas fueron un ambicioso registro de la mayoría de los

\footnotetext{
4 Destacan, en diferentes momentos, Edmundo Aray, Caupolicán Ovalles, Adriano González León, Salvador Garmendia, Rodolfo Izaguirre, entre otros.
} 


\section{Alejandro Cardozo Uzcátegui}

intelectuales jóvenes -más valiosos- de la década. Conformaron En Haa Jorge Nunes, Carlos Noguera, Argenis Daza Guevara, Aníbal Castillo, Víctor Salazar, Lubio Cardozo (histórico integrante del Partido Comunista de Venezuela), todos militantes de izquierda.

También había un deslinde, una traza política en la plástica, podemos demostrarla en el desencuentro ideológico entre la abstracción y la figuración vista en la crítica de Alejandro Otero al veredicto del Salón Oficial de 1957, donde los artistas figurativos gozaron del favor del jurado en menoscabo de los abstraccionistas. Otero Silva calificaba el abstraccionismo como arte evasivo: "Es una fórmula comprensible apenas para un cenáculo iniciado y minoritario, que niega al hombre en la tierra, que no quiere saber nada del pueblo ni de sus angustias, que pretende sustituir a la emoción artística por la apreciación cerebral de la obra." (Sainz, 2007: 59). En el realismo social destacaban en Venezuela César Rengifo y Gabriel Bracho, notorios militantes del PCV que desarrollaron el muralismo, siendo ellos junto con los mexicanos Rivera, Siqueiros y Orozco los referentes latinoamericanos de esa corriente. No podemos dejar en los papeles al Círculo del Pez Dorado (1962-1965), conformado por los pintores Jacobo Borges, Régulo Pérez (miembros del PCV) y Manuel Espinoza. Más adelante este grupo se trasformó en la Nueva Figuración, donde se suman Alirio Palacios y Alirio Rodríguez. Todos los anteriores miembros de diferentes organizaciones de izquierda, devenidas de las primeras divisiones del Partido Comunista.

Los grupos literarios posteriores (década de los ochenta) Tráfico y Guaire, durante el II Congreso de Escritores de la Lengua Española en 1981, reclamaron mayor compromiso revolucionario por parte de los escritores venezolanos de la década naciente de 1980, el proceso político de Nicaragua había captado la atención de estos grupos (Tráfico y Guaire) e inclusive sufrieron una ruptura respecto a apoyar o no la revolución sandinista.

Otro testimonio que transparenta esta circunstancia se lee en una entrevista al poeta Igor Barreto hecha por Karina Saiz Borgo: “En Tráfico había más ligazón política (...) allí había algo mucho más progresista, de izquierda, ligado a los íconos de esa cultura. En Guaire no (...) porque el fundador y aglutinador (...) de Guaire, que es Arraiz Lucca, jamás fue de izquierda y jamás se lo planteó" (Sainz, 2007: 124-130). Visto este panorama, Juan Liscano, prolífero crítico literario, resume en 1973 la deriva ideológica del aparato cultural venezolano y la naturaleza del régimen castrista, un contraste que debe señalarse:

Los años 61 y 62 fueron de intensa agitación política de izquierda. Los intelectuales activistas ya veían el derrumbamiento del sistema democrático y el ascenso al poder de las guerrillas triunfantes. El régimen parlamentario tuvo que enfrentarse al terrorismo urbano, a guerrillas rurales y a movimientos conspirativos castrenses. El presidente Rómulo Betancourt, se vio en la obligación de demostrar que un gobierno representativo era también capaz de defenderse, y ganó la partida. Pero a costa de duras batallas. El país quedó violentamente dividido. Si bien, en el momento de escribir este trabajo, el prestigio de la Revolución cubana ha disminuido mucho debido a su 
dependencia de la URSS, al personalismo de Castro y a la persecución de intelectuales, en cambio la voluntad revolucionaria ha derivado hacia las posiciones del Mayo francés, hacia el nihilismo del Poder Joven, hacia un repudio radical al sistema democrático burgués, pero también de la burocracia jerárquica soviética y de los partidos comunistas ortodoxos (Liscano, 1973: 126-127).

\title{
El aparato cultural venezolano de la Guerra Fría como medio de pacificación
}

El entonces expresidente Rómulo Betancourt fustigaba la situación - auténtica - de la cooptación cultural de la izquierda y, de hecho, en lugar de verla como una fortaleza de la democracia venezolana, sentía que era "un debilitamiento de los resortes democráticos":

\begin{abstract}
¿Cómo es posible que ese señor González León, autor de País Portátil (...) simplemente porque es marxista forme parte del jurado que otorgó el tercer premio del concurso literario "Rómulo Gallegos"? ¿Ah? Eso hay que decirlo y además impedirlo. (...) esto es un estatus reblandecido y hay gentes de mi propio partido que no tienen una idea clara de eso. ¿Cómo concibe usted la cantidad de millones que se han gastado en este país para glorificar fílmicamente a las guerrillas? ¡Ahora resulta que nuestros héroes son los guerrilleros! (...) Si estos señores se hubieran quedado tranquilos y dicen, bueno, reconocemos que nos equivocamos. Pero no, ahora magnifican sus actos y entonces resulta que lo que fue un crimen, no una equivocación, ahora es un acto heroico. Porque ¿cuánta gente mataron ellos, física y síquicamente?, ¿cuántos jóvenes quedaron frustrados, convertidos en alcohólicos y marihuaneros? [La entrevistadora responde] Pero es que si se retorna a este criterio duro, de aislarlos del sistema, de no permitirles manifestarse, ¿no ocurrirá un retroceso? Porque habrá otra vez respuesta a la represión y con reacciones violentas. ¿Hay derecho a que se repita la historia? Además, el partido socialcristiano Copei hizo la "Pacificación", dentro de la cual esa domesticación de las izquierdas fue un factor decisivo. Porque es un hecho que durante la presidencia del Dr. Rafael Caldera, fueron burocratizadas y neutralizadas en buena proporción (Freilich, 1981: 33-34).
\end{abstract}

El Estado venezolano entendió el hecho de que buena parte de la intelectualidad y las artes venezolanas no solo demostraban una sensibilidad creadora definitoria, sino como se ha visto a lo largo del artículo, una sensibilidad y cultura política de marcada tendencia hacia las izquierdas, la lucha social, urbana y armada. Por ello se crean espacios para distender -y no contener- la disputa política.

En 1966 se crea el Instituto Nacional de Cultura y Bellas Artes (Inciba) en 19665, desde donde se invitó, a cambio de la violencia, la oferta de una vida estimable, respetada en los terrenos de la actividad intelectual. Durante la presidencia de Simón Alberto Consalvi en el INCIBA comenzó a editarse la revista Imagen (fundada

\footnotetext{
5 Se decreta el 9 de marzo de 1960, el 8 de abril se sanciona el ejecútese del decreto. En octubre de 1964 el presidente Raúl Leoni nombró la junta directiva del Instituto.
} 


\section{Alejandro Cardozo Uzcátegui}

en 1967) como parte del espíritu político-cultural de tender puentes entre la izquierda intelectual y el joven Estado democrático. Diría Consalvi que en la dura refriega de los sesenta, se estableció entre el Estado democrático y la izquierda inserta en el aparato cultural venezolano, una dramática relación, donde la segunda pensaba que el Estado la estaba domesticando, comprando y desvinculando de la lucha armada y de la influencia cubana, "Sin embargo, como está escrito en Imagen, se hizo un gran esfuerzo para que todos tuvieran cabida y todos tuvieron cabida, incluso los más radicales de ellos (Sainz, 2007: 84-85).

Se crea el Centro de Estudios Latinoamericanos Rómulo Gallegos (Celarg) en 1974, que logró entrar en sintonía con los ensayos creadores de los grupos literarios que habían prometido la propuesta antisistémica global/nacional; con ello nace, también dentro del Celarg, los talleres de creación literaria. Así también nacieron la Casa Nacional de las Letras Andrés Bello y el monumental proyecto editorial Fundación Biblioteca Ayacucho. Sin embargo, sustituyó al Inciba con mayor eficacia en sus propósitos el Consejo Nacional de la Cultura (Conac), en 1975. Anota Juan Liscano, como presidente de la comisión y primer presidente del Consejo: "La ley del Conac, (...) propone el articulado y los principios tendientes a garantizar servicios culturales públicos sin someterlos a finalidades políticas parcializadas, lo cual queda avalado por la pluralidad ideológica" (1976: 954).

Más autoritarios y refractarios que el propio Estado, fueron los núcleos políticos de la izquierda radical que atacaron a escritores como Héctor Mujica por no permitir agresiones a la Revista Nacional de Cultura, o a Jesús Sanoja Hernández por haber presentado los libros de Ludovico Silva Marx y la alineación y el Anti-manual, obras revisionistas para los más doctrinarios. Asimismo Rodolfo Izaguirre fue reprochado por los "fundamentalistas" del Movimiento de Izquierda Revolucionaria y la revista Rocinante -dirigida por Edmundo Aray- por haber aceptado administrar la Cinemateca Nacional (Sainz, 2007).

\section{El Departamento de Estado de EEUU y el sistema cultural venezolano: la gira de William Faulkner}

Greg Barnhisel cita un reporte del viaje de Faulkner a Venezuela, hecho por un oficial del gobierno estadounidense llamado Hugh Jencks (hizo de intérprete del escritor durante la visita) en 1962, quien informa que:

The cultural leaders of Venezuela, many of whom are pre-disposed to take an anti-U.S. attitude on all international issues, include writers, artists, newspaper commentators (particularly those connected with El Nacional), educators and people in goverment. The group also includes many on-the-fencers. Its members tend to agree with the Communist tenet that the United States is grossly materialistic, with no cultural achievements. To bring a literary figure of the stature of Faulkner to Venezuela was an effective refutation of this view (Barhinsel, 2016, 132). 


\section{Una historia cultural de la Guerra Fría en El Caribe: análisis comparado del nacimiento de los aparatos culturales de Cuba y Venezuela (1960-1980)}

Para el analista del Departamento de Estado Hugh Jenks, hubo que invitar al gran novelista (y premio nobel de 1949) William Faulkner a Venezuela para refutar el "principio comunista" de que EEUU es una nación extremadamente materialista y sin logros culturales; la deslegitimación cultural del mundo capitalista, había sido uno de los aspectos del correlato del mundo socialista, para doblar la matriz de opinión en la Guerra Fría cultural, desdiciendo los hitos culturales de Estados Unidos.

Es menester puntualizar algunos elementos del viaje de William Faulkner durante dos semanas a Venezuela, en abril de 1961, promovido por el Departamento de Estado para lograr "un mejor entendimiento cultural" entre ambas naciones. La fama literaria de Faulkner tuvo también relación con un plan de Guerra Fría cultural donde se impulsaba el modernismo angloamericano como un instrumento del anticomunismo. Justamente, el primer viaje del novelista fue en agosto de 1954, menos de un mes había transcurrido de la intervención en Guatemala y el derrocamiento de Jacobo Arbenz (Schwartz, 1990).

En su tour venezolano Faulkner se reunió con el presidente Betancourt, Rómulo Gallegos, Uslar Pietri, Juan Bosch y Arturo Croce. Fue condecorado por la Orden Andrés Bello, preparó y dijo su discurso de orden en castellano. Faulkner entendió su misión como un servicio político-cultural a su país: "había esperado que la nueva administración [Kennedy] ya hubiese elaborado para aquel tiempo una política exterior. Entonces amateurs como yo (los reacios) no necesitaríamos ser enviados al frente"6 (Padilla, 2018). Un informe oficial de la United States Information Agency decía que "estuvieron constantemente junto a él [Faulkner] durante su estadía para evitar cualquier incidente mayor y toda cobertura de prensa desfavorable que pudiesen realizar los periódicos comunistas" (Oakley, 2004, 413).

Como acotaría John Campbell, el viaje no logró cumplir las máximas expectativas, y el gasto de la inversión no se vio cumplido con los resultados. Recuérdese que es el mismo año de la invasión de Bahía de Cochinos (al día siguiente del retorno de Faulkner ocurrió la operación "Mangoose"), apenas tres años antes intentan linchar en Caracas al vicepresidente Nixon, la lucha armada se organizaba en Venezuela, y apenas comenzaba el giro JFK en política exterior, es decir, el aparato cultural de izquierda en Venezuela estaba en una situación vigorosa, y la visita de Faulkner no resultó en nada para ablandar la percepción que había de EEUU en los nichos fuertes de la cultura política venezolana.

En miras de mejorar aquello, se estableció, entre otras directrices -dentro del formato del poder blando y la gran estrategia cultural estadounidense- la Fundación

\footnotetext{
${ }^{6}$ Sobre los motivos de la visita del novelista a Venezuela se plantea dos versiones: al llegar Kennedy a la presidencia de Estados Unidos, le solicitó al novelista que fuera su embajador cultural, una especie de pica en Flandes dentro de su proyecto político para América Latina de la Alianza para el Progreso y, particularmente para Venezuela, donde se había insultado y escupido al Vicepresidente Nixon durante la administración Eisenhower tres años antes. La otra versión, de Edgard Mondolfi Gudat, afirma que la iniciativa vino del Gobierno de Venezuela presidido por Rómulo Betancourt. La invitación fue hecha a través de la Asociación Norteamericana de Venezuela con el plácet del gobierno de Kennedy.
} 


\section{Alejandro Cardozo Uzcátegui}

Faulkner, donde se premia en 1965 al escritor venezolano Ramón Díaz Sánchez por la novela Cumboto, donde, entre otras cosas, prometía la edición de la novela al inglés para ser distribuida entre el público estadounidense, a pesar de los prejuicios editoriales en EEUU por el tratamiento que el autor daba al tema de las negritudes en Venezuela. ${ }^{7}$

\section{Conclusión}

En el marco de la Guerra Fría cultural, el Estado venezolano optó por la apertura de espacios controlados para la disidencia ideológica de la mayor parte de sus intelectuales y artistas, creadores que reivindicaron en su obra, el derrumbamiento del Estado que los beneficiaba en muchos sentidos materiales y políticos. El Estado cubano, por otro lado, auspició la creación de un aparato cultural pero no para la pacificación de sus intelectuales, sino para la promoción de la revolución, en toda medida, Cuba no toleró la disidencia contra el sistema patrocinador -en Venezuela sí- y paradójicamente el aparato cultural venezolano terminó siendo otro promotor más de la revolución cubana y sus diferentes formas de expresión ideológica; apoyaron la guerrilla revolucionaria venezolana y luego formaron parte, en gran escala, del aparato cultural del mundo socialista.

En Cuba el aparato cultural nació desde la revolución para estancarlo en la conservación del régimen del partido, y en Venezuela nació desde el poder establecido pero se le obsequió a intelectuales, artistas y promotores culturales de izquierda para que lo construyeran y desarrollaran en aras de la "Paz democrática", y por la inclusión ideológica de la democracia. Fueron dos líneas diferentes, de la revolución para solidificar el poder establecido (Cuba), y desde el poder establecido para promover una estética revolucionaria de izquierda contra el mismo poder que le dio vida al aparato cultural (Venezuela).

\section{Bibliografía}

"Alfonso,

Paco".

(2020).

EcuRed.

Recuperado

de https://www.ecured.cu/Paco Alfonso

"Marinello Vidaurreta, Juan". (2020). EcuRed. Recuperado de https://www.ecured.cu/Juan Marinello\#Trayectoria revolucionaria

"Núñez Jiménez, Antorio". (2020). EcuRed. Recuperado de https://www.ecured.cu/Antonio N\%C3\%BA\%C3\%B1ez Jim\%C3\%A9nez

\footnotetext{
7 Termina editándose por una iniciativa de la Universidad de Texas, dos años más tarde y con otra traducción alternativa a la primera propuesta.
} 
Una historia cultural de la Guerra Fría en El Caribe: análisis comparado del
nacimiento de los aparatos culturales de Cuba y Venezuela (1960-1980)

Alarcón de Quesada, R. (2009). "Vicentina Antuña: maestra, madre, compañera". Revolución y Cultura, 5 (6), 13-15.

Antuña Tavío, V. (1985). "Palabras pronunciadas por la Dra. Vicentina Antuña el 8 de noviembre de 1984, en el acto de constitución de la Cátedra por la Lectura «Camila Heríquez Ureña»". Revista Universidad de La Habana, 220-221.

Aray, E. (2017). Tierra roja, tierra negra. Caracas: El perro y la rana.

Arráiz, R. (2003). El coro de las voces solitarias. Una historia de la poesía venezolana. Caracas: Eclepsidra.

Barhinsel, G. (2016). Cold War Modernists: Art, Literature, and American Cultural Diplomacy, Nueva York: Columbia University Press.

Calduch, R. (1993). Dinámica de la Sociedad Internacional. Madrid: Centro de Estudios Ramón Areces.

Calles-Santillana, J. (2013). “La plusvalía ideológica: Ludovico Silva y el análisis crítico de la comunicación en Latinoamérica". Razón y Palabra. Recuperado de http://www.razonypalabra.org.mx/N/N82/V82/47 Calles V82.pdf

Castro, F. (1961). "Palabras a los intelectuales" Recuperado de http://www.uneac.org.cu/sites/default/files/pdf/publicaciones/boletin se dice c ubano no.9.pdf

Calzadilla, J. (1995). “El Techo de la Ballena”. Aleph (8) 9, 24-28.

Cancio, W. (2008). "Recuerdan brillante trayectoria de la revista Bohemia en su centenario". El Nuevo Herald. Recuperado de https://www.elnuevoherald.com/ultimas-noticias/article1932306.html

Cardozo, L. (1976). Philobiblion. Mérida: Universidad de Los Andes.

Cayuela, R. (2006). “Entrevista con Carlos Franqui”. Letras Libres. Recuperado de https://www.letraslibres.com/mexico/entrevista-carlos-franqui

Chaffy, W y G. Prevost. (1992). Cuba. A Different America. Boston: Rowman and Littefield Publishers, Inc.

Chao, E. (2019). Marxists at the Gate. Washington DC: Dupont Circle Edition

Domingo, J. (2007). Una mirada a la vida intelectual cubana: (1940-1950). Málaga: Diputación de Málaga.

Farber, S. (2006). The Origins of the Cuban Revolution Reconsidered. N.C.: The University of North Carolina. 


\section{Alejandro Cardozo Uzcátegui}

Fontana J. (2013). Por el bien del imperio. Una historia del mundo desde 1945. Barcelona: Pasado \& Presente.

Freilich, A. (1981). La venedemocracia. Caracas: Monte Ávila Editores.

García Marañón, F. (2018). Myrna Torres y Ernesto Guevara en Guatemala. Tlamelaua, 12(45), 252-257. Recuperado en 06 de abril de 2020, Recuperado de http://www.scielo.org.mx/scielo.php?script=sci arttext\&pid=S187069162018000200252\&lng=es\&tlng=es.

Guédez, J. (2003). “Hablemos de poesía” fuete de origen digital, última captura hecha el 12 de abril de 2018. Recuperado de migueleguedez.wordpress.com/2009/02/05/entrevista-a-jesus-enrique-guedez2003/

Guzmán, J. (2012). “Actores gubernamentales de la política cultural cubana entre 1949 y 1961”. Revista Latinoamericana de Ciencias Sociales, Niñez y Juventud, 10 (1), 257-270.

Káiser, P. (2011). “Cine venezolano”. Diccionario del Cine Iberoamericano. España, Portugal y América. SGAE.

Lambert, L. (1996). "Presencia e imagen de la mujer en la revista cubana Bohemia”. América: Cahiers du CRICCAL, 15 (16) 133-146.

Lanza, E. (2018). "Informe especial sobre la situación de la libertad de expresión en Cuba". Relatoría Especial para la Libertad de Expresión de la Comisión Interamericana de Derechos Humanos. Organización de Estados Americanos. Recuperado de http://www.oas.org/es/cidh/expresion/docs/informes/Cubaes.pdf

Liscano, J. (1973). Panorama de la literatura venezolana actual. Caracas: Publicaciones Españolas de Caracas.

Liscano, J. (1976). "50 años de Cultura en Venezuela”. Venezuela moderna. Medio siglo de historia 1926-1976. Caracas: Fund. Eugenio Mendoza.

Massiani, Felipe. (1977). La política cultural en Venezuela. París: Unesco.

Miranda, L. (1984). Lázaro Peña: capitán de la clase obrera cubana. La Habana: Editorial De Ciencias Sociales.

Miranda-Cancela, E. (2019). "Vicentina en la memoria”. Revolución y Cultura, 5-6, 1620.

Nye, J. (2003). La paradoja del poder norteamericano. Madrid: Taurus. 
Una historia cultural de la Guerra Fría en El Caribe: análisis comparado del
nacimiento de los aparatos culturales de Cuba y Venezuela (1960-1980)

Oakley, H. (2004). "Foulkner and the Cold War" en Smith, Jon, Cohn, Deborah (eds.) Look. Away!... Away! The U.S. South in New World Studies. Durham: Duke University Press.

Padilla, F. (2013). "Faulkner: el granjero en Venezuela”. Revistacrónica, Recuperado de http://www.revistacoronica.com/2013/08/faulkner-en-venezuela.html

Pino, M. (2006). "El Semanario Marcha de Uruguay: una genealogía de la crítica de la cultura en América Latina”. Revista de Crítica Latinoamericana, 56, 141-156.

Quevedo, M. (2019). “Carta de Despedida de Miguel Ángel Quevedo”. América 2.1 Recuperado de https://americanuestra.com/carta-de-despedida-de-miguel-angelquevedo/

Quintero Heredia, J. (2002). Fulguración del espacio: Letras e imaginario institucional de la Revolución Cubana (1960-1971). Buenos Aires: Beatriz Viterbo Editora.

Rey, J. (1991). "La democracia venezolana y la crisis del sistema populista de conciliación”. Revista de Estudios Políticos, 74, 533-578.

Rojas, R. (2015). Historia mínima de la Revolución cubana. México: El Colegio de México.

Rojas, R. (2018). La polis literaria. El Boom, la Revolución y otras polémicas de la Guerra Fría. Barcelona: Taurus.

Ruiz, F. (2003). Otra grieta en la pared: Informe y testimonios de la Nueva Prensa Cubana. La Habana: Sociedad de Periodistas Manuel Márquez.

Sainz, K. (2007). Cuatro reportajes, dos décadas, una historia: Tráfico y Guaire, el país y sus intelectuales. Caracas: Fondo para la Cultura Urbana.

Schwartz, L. (1990). Creating Faulkner's Reputation: The Politics of Modern Literary Criticism. Tennessee: University of Tennessee Press.

Slack, T. y Whitson, D. (1988). "The Place of Sport in Cuba's Foreign Relations". International Journal: Canada's Journal of Global Policy Analysis. https://doi.org/10.1177/002070208804300404

Torreira, R. (2005). La iglesia católica en la primera oleada migratoria cubana. Santiago de Cuba: Editora política.

Torricella, R. (2012). "Ernesto Montaner... Un editorial que hoy tiene vigencia”. Zoe Valdés https://zoevaldes.net/2012/12/22/ernesto-montaner-un-editorial-quehoy-tiene-vigencia-por-roberto-torricella/

Zubok, V. (2008). Un imperio fallido. La Unión Soviética durante la Guerra Fría. Barcelona: Crítica. 


\section{Alejandro Cardozo Uzcátegui}

\section{Documentos}

Braddock, Daniel M (1959) "Despatch From the Embassy in Cuba to the Department of State", https://history.state.gov/historicaldocuments/frus1958-60v06/d278

CIA (1965) Whereabouts Of David Salvador Manso, Founder Ff 30th Of November Revolutionary

Movement, https://www.cia.gov/library/readingroom/docs/DOC_0005530646.pdf

Department of State Executive Secretariat Files (1959). Lot 63D351: NSC 64 Series. https://history.state.gov/historicaldocuments/frus1950v06/d480

Stephansky B. (1959). Foreign Relations of the United States, 1958-1960, Cuba, vol. VI (299), "Memorandum From Benjamin Stephansky of the Office of Inter-American Regional Political Affairs to the Deputy Assistant Secretary of State for InterAmerican Affairs". https://history.state.gov/historicaldocuments/frus1958$\underline{60 v 06 / d 299}$

Recibido: 11/08/2020

Evaluado: 20/09/2020

Versión Final: 15/01/2021 